\title{
A general algorithm for numerical evaluation of nearly singular integrals over high-order geometry elements in 3D BEM
}

\author{
Y. M. Zhang ${ }^{1,2}$, X. C. Li ${ }^{1}$, V. Sladek ${ }^{3}$ \& J. Sladek ${ }^{3}$ \\ ${ }^{1}$ Institude of Applied Mathematics, \\ Shandong University of Technology, P. R. China \\ ${ }^{2}$ State Key Laboratory of Structural Analysis for Industrial Equipment, \\ Dalian University of Technology, P. R. China \\ ${ }^{3}$ Slovak Academy of Sciences, Institute of Construction and Architecture, \\ Bratislava, Slovakia
}

\begin{abstract}
The accurate evaluation of nearly singular boundary integrals is an important issue in BEM. Several techniques have been developed in recent years, with various degree of success, but it should be stressed that the boundary geometry is depicted essentially by using lower-order elements in those approaches. However, a high level of the geometry approximation in BEM is desired in many applications, and the usage of high-order elements can meet this requirement. In this study, we propose a general methodology for computation of the nearly singular integrals with using high-order surface elements in 3D BEM. Using two benchmark solutions for potential problems we demonstrate the high efficiency and the stability of the proposed scheme, even when the internal point is very close to the boundary.

Keywords: BEM, nearly singular integrals, transformation, high-order geometry elements, potential problem.
\end{abstract}

\section{Introduction}

The BEM as an important technique has been widely used in many areas, but the accuracy of its calculation depends heavily on the accuracy and efficiency of the computation of integrals with singular and nearly singular kernels. These 
kernels are weakly singular, strongly singular, or even hypersingular functions when the collocation point belongs to the integration elements, and many effective methods have been developed to handle them [1-3]. Another important issue is the integration of the kernels for the collocation points which are close to but not on the integration element. Then, the considered integrals, although regular in the sense of mathematics, are termed nearly weak singular, nearly strong singular and nearly hypersingular integrals since they exhibit similar characteristics of the singularity and generally cannot be calculated accurately by standard integration quadratures. This is so-called boundary layer effect in BEM.

In the past decades, tremendous effort has been devoted to derive convenient integral forms or sophisticated computational techniques for calculating the nearly singular integrals. These proposed methods can be divided on the whole into two categories: "indirect algorithms" [4, 5] and "direct algorithms" [6-19]. The indirect algorithms consist in indirect calculation or in avoiding calculation of the nearly singular integrals by establishing new regularized BIEs using the rigid-body displacement idea or other global regularization methods [4, 5]. The direct algorithms consist in calculation or approximation of the nearly singular integrals directly. They usually include interval subdivision [6], special Gaussian quadrature [7], analytical or semi-analytical [8,9] and transformation methods [10-19]. At present, the most popularity of the "direct algorithms" are various non-linear transformations, for example, the cubic polynomial transformation [10], the bi-cubic transformation [11], the sigmoidal transformation [12], the semi-sigmoidal transformation [13], the coordinate optimal transformations [14], the degenerate mapping method [15], the rational transformation [16], the distance transformation [17], the sinh trans-formation [18], and the exponential trans-formation [19], etc.

Although great progresses have been achieved for each of the above methods, it should be pointed out that the geometry of the boundary element is depicted essentially by using lower-order shape functions when nearly singular integrals need to be calculated. In fact, to the authors' best knowledge, no work is found in the literature that can be used to calculate the nearly singular integrals occurring on high-order geometry elements in 3D BEM effectively. However, the usage of high-order geometry elements for efficient evaluation of the nearly singular integrals is necessary in many cases. Clearly, the advantage of using the high-order geometry elements in complex geometrical domains does not concern only its power to achieve higher calculation accuracy, but even with a small number of computational elements. More crucial, computational models of thin structure problems demand a higher level of geometry approximation, and the usage of high-order geometry in computational model can meet this requirement. If the boundary geometry is depicted by using plane elements, the actual geometry of considered domain cannot be described faithfully, and thus lowerorder geometry approximation will fail to yield reliable results. In order to avoid such phenomena, very fine meshes must be used in these situations, but this yields too much preprocessing and prolongs the CPU time. In addition, a great number of elements will produce a lot of artificial corners. This is fatal to many engineering problems. 
On the other hand, it is well-known that the efficient estimation of nearly singular integrals over high-order geometry elements is a very difficult problem. When the geometry of the boundary element is approximated by using high order curved surface elements-usually of the second order, the Jacobian $J\left(\xi_{1}, \xi_{2}\right)$ is not a constant but an irrational function, where $\xi_{1}, \xi_{2}$ are the dimensionless coordinates. The distance $r$ between the field point and the source point is an irrational function of the type $\sqrt{p\left(\xi_{1}, \xi_{2}\right)}$, where $p\left(\xi_{1}, \xi_{2}\right)$ is the high order polynomial. Thus, the integrands in boundary integrals become rather complex to treat the nearly singular integrals.

In this study, a general strategy is proposed for calculation of the nearly singular integrals occurring on high-order curved surface elements in 3D BEM. The paper is organized as follows: The regularized BIEs for indirect unknowns with excluding the CPV and HFP integrals are presented in Section 2. Then, in Section 3, the expression of the nearly singular integrals over the eight-node second-order quadrilateral surface elements is investigated, including the determination of the projection point and construction of the distance function. Section 4 introduces an exponential transformation to remove or reduce the near singularities of integrands for considered integrals. In Section 5, the accuracy and stability of the proposed scheme are tested in two 3D potential examples with known benchmark solutions. Finally, the conclusions are provided in Section 6.

\section{Regularized boundary integral equations (RBIEs)}

In this paper, we always assume that $\Omega$ is a bounded domain in $R^{3}, \Omega^{c}$ is its open complement, and $\Gamma$ denotes the common boundary. $\boldsymbol{t}(\boldsymbol{x})$ and $\boldsymbol{n}(\boldsymbol{x})$ are the unit tangent and outward normal vectors on $\Gamma$ to the domain $\Omega$ at the point $\boldsymbol{x}$, respectively. With omitting the body sources in potential problems, the regularized BIEs with indirect unknowns on $\hat{\Omega}$ can be expressed as

$$
\begin{gathered}
u(\boldsymbol{y})=\int_{\Gamma} \phi(\boldsymbol{x}) u^{*}(\boldsymbol{x}, \boldsymbol{y}) d \Gamma, \boldsymbol{y} \in \Gamma \\
\frac{\partial u(\boldsymbol{y})}{\partial \hat{\boldsymbol{n}}(\boldsymbol{y})}=\hat{S} \phi(\boldsymbol{y})+\int_{\Gamma}[\phi(\boldsymbol{x})-\phi(\boldsymbol{y})] \frac{\partial u^{*}(\boldsymbol{x}, \boldsymbol{y})}{\partial \hat{\boldsymbol{n}}(\boldsymbol{y})} d \Gamma \\
+\phi(\boldsymbol{y}) \int_{\Gamma}\left[\frac{\partial u^{*}(\boldsymbol{x}, \boldsymbol{y})}{\partial \hat{\boldsymbol{n}}(\boldsymbol{y})}+\frac{\partial u^{*}(\boldsymbol{x}, \boldsymbol{y})}{\partial \hat{\boldsymbol{n}}(\boldsymbol{x})}\right] d \Gamma, \boldsymbol{y} \in \Gamma
\end{gathered}
$$

For the internal point $\boldsymbol{y}$, the integral equations can be written as

$$
\begin{gathered}
u(\boldsymbol{y})=\int_{\Gamma} \phi(\boldsymbol{x}) u^{*}(\boldsymbol{x}, \boldsymbol{y}) d \Gamma_{x}, \boldsymbol{y} \in \hat{\boldsymbol{\Omega}} \\
\nabla_{\boldsymbol{y}} u(\boldsymbol{y})=\int_{\Gamma} \phi(\boldsymbol{x}) \nabla_{y} u^{*}(\boldsymbol{x}, \boldsymbol{y}) d \Gamma_{x}, \boldsymbol{y} \in \hat{\boldsymbol{\Omega}}
\end{gathered}
$$


In eqns (1)-(4), $\phi(\boldsymbol{x})$ is the density function to be determined; $u^{*}(\boldsymbol{x}, \boldsymbol{y})$ denotes the Kelvin fundamental solution. For interior problems, $\hat{\Omega}=\Omega, \hat{S}=1$, $\hat{\boldsymbol{n}}(\boldsymbol{x})$ is the unit outward normal vectors on $\Gamma$ to domain $\Omega$ at point $\boldsymbol{x}$. For exterior problems, $\hat{\Omega}=\Omega^{c}, \hat{S}=0, \hat{\boldsymbol{n}}(\boldsymbol{x})$ is the unit outward normal vectors on $\Gamma$ to domain $\Omega^{c}$ at point $\boldsymbol{x}$.

For the discretized form of eqns (3) and (4), when the field point $\boldsymbol{y}$ is very close to the integration elements $\Gamma_{e}$, the integrals in discretized eqns (3)-(4) are nearly singular. These nearly singular integrals can be expressed as

$$
\mathrm{I}=\int_{\Gamma_{e}} \frac{f(x, y)}{r^{\alpha}} d \Gamma
$$

where $r=\|\boldsymbol{x}-\boldsymbol{y}\|_{2}, \alpha>0$ is a real constant, and $f(x, y)$ denotes a well-behaved function.

\section{Nearly singular integrals on paraboloidal surface elements}

In this paper, the geometry segment is modeled by a continuous paraboloidal element, which has eight knots, namely, the boundary geometry is approximated by the piecewise continuous eight-node second-order quadrilateral surface elements, while the distribution of the boundary quantities over each of these segments is approximated using discontinuous elements, eight nodes of which are located away from the edges of the element.

Assume $\boldsymbol{x}^{j}=\left(x_{1}^{j}, x_{2}^{j}, x_{3}^{j}\right), j=1, \cdots, 8$ are the eight knots of the segment $\Gamma_{j}$, then Cartesian coordinates of the points on the element $\Gamma_{j}$ can be interpolated as

$$
x_{k}\left(\xi_{1}, \xi_{2}\right)=\sum_{j=1}^{8} N_{j}\left(\xi_{1}, \xi_{2}\right) x_{k}^{j}, \quad k=1,2,3
$$

where

$$
\begin{aligned}
& N_{1}\left(\xi_{1}, \xi_{2}\right)=\frac{1}{4}\left(1-\xi_{1}\right)\left(1-\xi_{2}\right)\left(-\xi_{1}-\xi_{2}-1\right), N_{2}\left(\xi_{1}, \xi_{2}\right)=\frac{1}{4}\left(1+\xi_{1}\right)\left(1-\xi_{2}\right)\left(\xi_{1}-\xi_{2}-1\right), \\
& N_{3}\left(\xi_{1}, \xi_{2}\right)=\frac{1}{4}\left(1+\xi_{1}\right)\left(1+\xi_{2}\right)\left(\xi_{1}+\xi_{2}-1\right), N_{4}\left(\xi_{1}, \xi_{2}\right)=\frac{1}{4}\left(1-\xi_{1}\right)\left(1+\xi_{2}\right)\left(-\xi_{1}+\xi_{2}-1\right), \\
& N_{5}\left(\xi_{1}, \xi_{2}\right)=\frac{1}{2}\left(1-\xi_{1}^{2}\right)\left(1-\xi_{2}\right), N_{6}\left(\xi_{1}, \xi_{2}\right)=\frac{1}{2}\left(1+\xi_{1}\right)\left(1-\xi_{2}^{2}\right), \\
& N_{7}\left(\xi_{1}, \xi_{2}\right)=\frac{1}{2}\left(1+\xi_{2}\right)\left(1-\xi_{1}^{2}\right), N_{8}\left(\xi_{1}, \xi_{2}\right)=\frac{1}{2}\left(1-\xi_{1}\right)\left(1-\xi_{2}^{2}\right), \quad-1 \leq \xi_{1} \leq 1,-1 \leq \xi_{2} \leq 1
\end{aligned}
$$

\subsection{Determination of the projection point}

The minimum distance $d$ from the field point $y$ to the integration element $\Gamma_{e}$ is defined as the length $\left|\boldsymbol{y}-\boldsymbol{x}^{p}\right|$, where $\boldsymbol{x}^{p}$ is the projection point of $\boldsymbol{y}$ onto integration element $\Gamma_{e}$. Letting $\left(\eta_{1}, \eta_{2}\right)$ be the local coordinates of the 
projection point $\boldsymbol{x}^{p}$, i.e. $\boldsymbol{x}^{p}=\left(x_{1}\left(\eta_{1}, \eta_{2}\right), x_{2}\left(\eta_{1}, \eta_{2}\right), x_{3}\left(\eta_{1}, \eta_{2}\right)\right)$, then $\eta_{1}, \eta_{2}$ are the real roots of the following equation

$$
\left\{\begin{array}{l}
{\left[x_{i}\left(\eta_{1}, \eta_{2}\right)-y_{i}\right] \frac{\partial x_{i}}{\partial \xi_{1}}=0} \\
{\left[x_{i}\left(\eta_{1}, \eta_{2}\right)-y_{i}\right] \frac{\partial x_{i}}{\partial \xi_{2}}=0}
\end{array}, \quad i=1,2,3\right.
$$

in which the summation convention is used, and $\frac{\partial x_{i}}{\partial \xi_{k}}=\left.\frac{\partial x_{i}}{\partial \xi_{k}}\right|_{\substack{\xi_{1}=\eta_{1} \\ \xi_{2}=\eta_{2}}}, k=1,2$. These assumptions will be applied also in what follows unless specified others.

If the field point $\boldsymbol{y}$ is sufficiently close to the boundary $\Gamma$, then $\boldsymbol{x}^{p}$ is inside the integration element, and eqn. (8) has a pair of the unique real roots $\left(\eta_{1}, \eta_{2}\right) \in[-1,1] \times[-1,1]$. The real roots $\eta_{1}, \eta_{2}$ can be evaluated numerically by using the Newton's method. Setting

$$
f_{1}\left(\eta_{1}, \eta_{2}\right)=\left[x_{i}\left(\eta_{1}, \eta_{2}\right)-y_{i}\right] \frac{\partial x_{i}}{\partial \xi_{1}}, f_{2}\left(\eta_{1}, \eta_{2}\right)=\left[x_{i}\left(\eta_{1}, \eta_{2}\right)-y_{i}\right] \frac{\partial x_{i}}{\partial \xi_{2}}
$$

The formula of the Newton method can be expressed as

$$
F^{\prime}\left(\eta^{(k)}\right) \Delta \eta^{(k)}=-F\left(\eta^{(k)}\right)
$$

where $\Delta \eta^{(k)}=\eta^{(k+1)}-\eta^{(k)}, \eta^{(k)}=\left(\eta_{1}{ }^{(k)}, \eta_{2}{ }^{(k)}\right)^{\mathrm{T}}, \eta^{(k+1)}=\left(\eta_{1}^{(k+1)}, \eta_{2}{ }^{(k+1)}\right)^{\mathrm{T}}$

$$
F\left(\eta^{(k)}\right)=\left[\begin{array}{c}
f_{1}\left(\eta_{1}^{(k)}, \eta_{2}{ }^{(k)}\right) \\
f_{2}\left(\eta_{1}{ }^{(k)}, \eta_{2}{ }^{(k)}\right)
\end{array}\right], F^{\prime}\left(\eta^{(k)}\right)=\left[\begin{array}{cc}
\frac{\partial f_{1}}{\partial \eta_{1}} & \frac{\partial f_{1}}{\partial \eta_{2}} \\
\frac{\partial f_{2}}{\partial \eta_{1}} & \frac{\partial f_{2}}{\partial \eta_{2}}
\end{array}\right]_{\eta=\eta^{(k)}}
$$

here $\frac{\partial f_{j}}{\partial \eta_{m}}=\sum_{i}^{3}\left(\frac{\partial x_{i}}{\partial \xi_{m}} \frac{\partial x_{i}}{\partial \xi_{j}}+\left[x_{i}\left(\eta_{1}, \eta_{2}\right)-y_{i}\right] \frac{\partial^{2} x_{i}}{\partial \xi_{j} \partial \xi_{m}}\right), j, m=1,2$

\subsection{Form of distance function $r^{2}$}

$x_{k}\left(\xi_{1}, \xi_{2}\right)$ can be expressed as

$$
x_{k}\left(\xi_{1}, \xi_{2}\right)=x_{k}\left(\eta_{1}, \eta_{2}\right)+\left(\xi_{\alpha}-\eta_{\alpha}\right) \frac{\partial x_{k}}{\partial \xi_{\alpha}}+\frac{1}{2}\left(\xi_{\alpha}-\eta_{\alpha}\right)\left(\xi_{\beta}-\eta_{\beta}\right) \frac{\partial^{2} x_{k}}{\partial \xi_{\alpha} \partial \xi_{\beta}}
$$

thus, we have 


$$
\begin{aligned}
r^{2}\left(\xi_{1}, \xi_{2}\right)= & {\left[x_{k}\left(\xi_{1}, \xi_{2}\right)-y_{k}\right]\left[x_{k}\left(\xi_{1}, \xi_{2}\right)-y_{k}\right] } \\
= & d^{2}+\left(\xi_{\alpha}-\eta_{\alpha}\right) \tilde{g}_{\alpha}+\left(\xi_{\alpha}-\eta_{\alpha}\right)\left(\xi_{\beta}-\eta_{\beta}\right) \tilde{g}_{\alpha \beta} \\
& +\left(\xi_{\alpha}-\eta_{\alpha}\right)\left(\xi_{\beta}-\eta_{\beta}\right)\left(\xi_{\gamma}-\eta_{\gamma}\right) \tilde{g}_{\alpha \beta \gamma} \\
& +\left(\xi_{\alpha}-\eta_{\alpha}\right)\left(\xi_{\beta}-\eta_{\beta}\right)\left(\xi_{\gamma}-\eta_{\gamma}\right)\left(\xi_{\mu}-\eta_{\mu}\right) \tilde{g}_{\alpha \beta \gamma \mu}
\end{aligned}
$$

where the summation rule is applied with respect to the Latin indices (taken from the range 1,2,3) and Greek indices (taken from the range 1,2)

$$
\begin{gathered}
d^{2}=\left(y_{k}-x_{k}^{p}\right)\left(y_{k}-x_{k}^{p}\right) \\
\tilde{g}_{\alpha}=2\left(y_{k}-x_{k}^{p}\right) x_{k, \alpha}, x_{k, \alpha}=\left.\frac{\partial x_{k}}{\partial \xi_{\alpha}}\right|_{\substack{\xi_{1}=\eta_{1} \\
\xi_{2}=\eta_{2}}} \\
\tilde{g}_{\alpha \beta}=\left(y_{k}-x_{k}^{p}\right) x_{k, \alpha \beta}+x_{k, \alpha} x_{k, \beta}=\tilde{g}_{\beta \alpha} \\
\tilde{g}_{\alpha \beta \gamma}=x_{k, \alpha \beta} x_{k, \gamma}=\tilde{g}_{\beta \alpha \gamma}, \tilde{g}_{\alpha \beta \gamma \mu}=\frac{1}{4} x_{k, \alpha \beta} x_{k, \gamma \mu}=\tilde{g}_{\beta \alpha \gamma \mu}
\end{gathered}
$$

Recall that $\tilde{g}_{\alpha} \equiv 0$, since $\left(y_{k}-x_{k}^{p}\right)$ is orthogonal to the element and $x_{k, \alpha}$ is tangential to the element at the projection point $\boldsymbol{x}^{p}$. Thus, eqn. (11) can be rewritten as

$$
\begin{gathered}
r^{2}\left(\xi_{1}, \xi_{2}\right)=d^{2}+\left(\xi_{\alpha}-\eta_{\alpha}\right)\left(\xi_{\beta}-\eta_{\beta}\right) \hat{g}_{\alpha \beta} \\
=d^{2}+\left(\xi_{1}-\eta_{1}\right)^{2} g_{11}+\left(\xi_{2}-\eta_{2}\right)^{2} g_{22}+\left(\xi_{1}-\eta_{1}\right)\left(\xi_{2}-\eta_{2}\right) g_{12},
\end{gathered}
$$

where $\hat{g}_{\alpha \beta}=\tilde{g}_{\alpha \beta}+\left(\xi_{\gamma}-\eta_{\gamma}\right) \tilde{g}_{\alpha \beta \gamma}+\left(\xi_{\gamma}-\eta_{\gamma}\right)\left(\xi_{\mu}-\eta_{\mu}\right) \tilde{g}_{\alpha \beta \gamma \mu}$,

$$
g_{11}=\hat{g}_{11}, g_{22}=\hat{g}_{22}, g_{12}=\hat{g}_{12}+\hat{g}_{21}=2 \hat{g}_{12}
$$

\subsection{Nearly singular integrals on the second-order elements}

By some simple deductions and based on the expression form (13) of the distance function $r^{2}$, the nearly singular integrals in eqn. (7) would be reduced to the following form

$$
\mathrm{I}=\int_{0}^{B} \int_{0}^{A} \frac{f(x, y)}{\left[d^{2}+x^{2} g_{11}(x, y)+y^{2} g_{22}(x, y)+x y g_{12}(x, y)\right]^{\alpha}} d x d y
$$

where $A, B$ are two constants which are possibly different values in different integrals; $f(\cdot)$ is a regular function that consists of shape functions, Jacobian and terms which arise from talking the derivative of the integral kernels. 


\section{Variable transformation}

In this paper, the exponential transformation can be expressed as follows

$$
x=d\left(e^{m_{1}+m_{2} s}-1\right), y=d\left(e^{n_{1}+n_{2} t}-1\right), \quad-1 \leq s \leq 1,-1 \leq t \leq 1
$$

where $m_{1}=m_{2}=\frac{1}{2} \ln \left(1+\frac{A}{d}\right), \quad n_{1}=n_{2}=\frac{1}{2} \ln \left(1+\frac{B}{d}\right)$

Substituting (15) into eqn. (14), we obtain the following equation

$$
\mathrm{I}=\frac{1}{d^{2 \alpha-2}} \int_{-1}^{1} \int_{-1}^{1} \frac{f(s, t) m_{2} n_{2} e^{m_{1}+n_{1}+m_{2} s+n_{2} t}}{F(s, t)} d s d t
$$

where

$$
\begin{aligned}
& F(s, t)= \\
& {\left[1+\left(e^{m_{1}+m_{2} s}-1\right)^{2} g_{11}(x, y)+\left(e^{n_{1}+n_{2} t}-1\right)^{2} g_{22}(x, y)+\left(e^{m_{1}+m_{2} s}-1\right)\left(e^{n_{1}+n_{2} t}-1\right) g_{12}(x, y)\right]^{\alpha}}
\end{aligned}
$$

with $x$ and $y$ being given by eqn. (15).

\section{Numerical examples}

In this section, two benchmark numerical examples of 3D potential problems are examined to verify the methodology developed above.

The numerical solution accuracy at single computed point is assessed by means of the relative error defined by

$$
R E=\left|\frac{I_{\text {exa }}-I_{\text {num }}}{I_{\text {exa }}}\right|
$$

where $I_{\text {num }}$ and $I_{\text {exa }}$ denote the numerical and exact value at the evaluation points, respectively. Furthermore, the average relative error $(A R E)$ of the multiple computational results is defined by

$$
A R E=\frac{1}{N} \sum_{j=1}^{N}|R E(j)|
$$

where $R E(j)$ denotes the relative error at the jth evaluation point, $N$ is the number of the interior evaluation points.

In what follows, $d$ denotes the distance between the evaluation point and the integration boundary element.

Example 1 As shown in Fig. 1, this example concerns a three dimensional spherical structure with radius 1.0 . The prescribed temperature on the boundary is

$$
u=x_{1}^{2} / 2-x_{2}^{2} / 2+2 x_{1}+x_{3}
$$




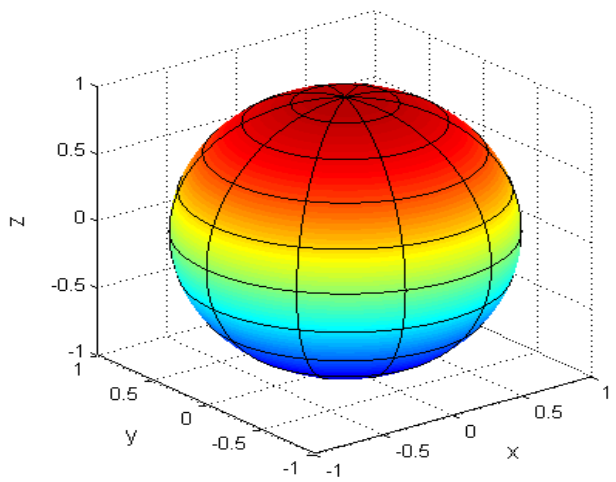

Figure 1: Discretization of a unit sphere with 100 second-order surface elements.

The spherical surface is divided into one hundred surface elements of the second-order, and the same type of discontinuous interpolation shape functions is adopted to approximate the boundary functions. The numerical solutions for the potentials $u$, and its derivatives $\partial u / \partial x_{1}$ (in the $x_{1}$ direction) at internal points are listed in Tables 1 and 2, respectively, hence we can see that when the evaluation points are not too close to the boundary, both the methods with and without transformation of the integration variables are effective and can give acceptable results. As the evaluation point approaches the boundary element of integration, i.e., when the distance of the internal point from the integration element is equal to or less than 0.001 , the results of the conventional method become less satisfactory. On the other hand, the results of the proposed method are still steady and satisfactory even when the distance of the evaluation point to the integration element reaches $1 \mathrm{E}-10$. This can be seen from the relative errors with respect to the exact solutions which are also shown in Tables 1 and 2 and demonstrate the efficiency and the usefulness of the developed algorithm.

Table 1: $\quad$ Potentials $u$ at internal points increasingly close to the boundary.

\begin{tabular}{|l|c|c|c|c|}
\hline \multirow{2}{*}{ Distance $d$} & \multirow{2}{*}{ Exact } & \multirow{2}{*}{ No transform } & \multicolumn{2}{c|}{ Present } \\
\cline { 4 - 5 } & & & Numerical & Relative error \\
\hline 0.1 & $0.220322 \mathrm{E}+01$ & $0.220359 \mathrm{E}+01$ & $0.220161 \mathrm{E}+01$ & $0.560426 \mathrm{E}-03$ \\
\hline 0.01 & $0.246821 \mathrm{E}+01$ & $0.247862 \mathrm{E}+01$ & $0.246719 \mathrm{E}+01$ & $0.412683 \mathrm{E}-03$ \\
\hline 0.001 & $0.249516 \mathrm{E}+01$ & $0.247213 \mathrm{E}+01$ & $0.249422 \mathrm{E}+01$ & $0.376121 \mathrm{E}-03$ \\
\hline 0.0001 & $0.249785 \mathrm{E}+01$ & $0.246942 \mathrm{E}+01$ & $0.249693 \mathrm{E}+01$ & $0.372091 \mathrm{E}-03$ \\
\hline 0.00001 & $0.249815 \mathrm{E}+01$ & $0.246912 \mathrm{E}+01$ & $0.249722 \mathrm{E}+01$ & $0.371644 \mathrm{E}-03$ \\
\hline 0.000001 & $0.249815 \mathrm{E}+01$ & $0.246909 \mathrm{E}+01$ & $0.249723 \mathrm{E}+01$ & $0.371656 \mathrm{E}-03$ \\
\hline 0.0000001 & $0.249777 \mathrm{E}+01$ & $0.246908 \mathrm{E}+01$ & $0.249680 \mathrm{E}+01$ & $0.371705 \mathrm{E}-03$ \\
\hline 0.00000001 & $0.249815 \mathrm{E}+01$ & $0.246908 \mathrm{E}+01$ & $0.249723 \mathrm{E}+01$ & $0.371886 \mathrm{E}-03$ \\
\hline 0.000000001 & $0.249815 \mathrm{E}+01$ & $0.249722 \mathrm{E}+01$ & $0.249680 \mathrm{E}+01$ & $0.372194 \mathrm{E}-03$ \\
\hline 0.0000000001 & $0.249815 \mathrm{E}+01$ & $0.246908 \mathrm{E}+01$ & $0.249722 \mathrm{E}+01$ & $0.372679 \mathrm{E}-03$ \\
\hline
\end{tabular}


Table 2: $\quad$ Potential derivatives $\partial u / \partial x_{1}$ at internal points increasingly close to the boundary.

\begin{tabular}{|l|c|l|c|c|}
\hline \multirow{2}{*}{ Distance $d$} & \multirow{2}{*}{ Exact } & \multirow{2}{*}{ No transform } & \multicolumn{2}{c|}{ Present } \\
\cline { 4 - 5 } & & & Numerical & Relative error \\
\hline 0.1 & $0.289926 \mathrm{E}+01$ & $0.291036 \mathrm{E}+01$ & $0.289666 \mathrm{E}+01$ & $0.895529 \mathrm{E}-03$ \\
\hline 0.01 & $0.298939 \mathrm{E}+01$ & $0.250781 \mathrm{E}+01$ & $0.299727 \mathrm{E}+01$ & $0.263732 \mathrm{E}-02$ \\
\hline 0.001 & $0.299839 \mathrm{E}+01$ & $-0.271481 \mathrm{E}+01$ & $0.300834 \mathrm{E}+01$ & $0.331862 \mathrm{E}-02$ \\
\hline 0.0001 & $0.299929 \mathrm{E}+01$ & $-0.329685 \mathrm{E}+01$ & $0.300945 \mathrm{E}+01$ & $0.339014 \mathrm{E}-02$ \\
\hline 0.00001 & $0.299938 \mathrm{E}+01$ & $-0.335008 \mathrm{E}+01$ & $0.300957 \mathrm{E}+01$ & $0.339736 \mathrm{E}-02$ \\
\hline 0.000001 & $0.299938 \mathrm{E}+01$ & $-0.335535 \mathrm{E}+01$ & $0.300958 \mathrm{E}+01$ & $0.339799 \mathrm{E}-02$ \\
\hline 0.0000001 & $0.299939 \mathrm{E}+01$ & $-0.335587 \mathrm{E}+01$ & $0.300957 \mathrm{E}+01$ & $0.339517 \mathrm{E}-02$ \\
\hline 0.00000001 & $0.299939 \mathrm{E}+01$ & $-0.335593 \mathrm{E}+01$ & $0.300945 \mathrm{E}+01$ & $0.335724 \mathrm{E}-02$ \\
\hline 0.000000001 & $0.299939 \mathrm{E}+01$ & $-0.335593 \mathrm{E}+01$ & $0.300938 \mathrm{E}+01$ & $0.333110 \mathrm{E}-02$ \\
\hline 0.0000000001 & $0.299939 \mathrm{E}+01$ & $-0.335593 \mathrm{E}+01$ & $0.300922 \mathrm{E}+01$ & $0.328025 \mathrm{E}-02$ \\
\hline
\end{tabular}

Example 2 The second example concerns a problem in a torus centered at origin, as shown in Fig. 2, with the exterior radius and interior radius being $R=3$ and $r=1$, respectively. The parametric equation of the boundary surface is

$$
\begin{aligned}
& x_{1}=(R+r \cos \theta) \cos \varphi, x_{2}=(R+r \cos \theta) \sin \varphi, x_{3}=r \sin \theta \\
& 0 \leq \theta \leq 2 \pi, 0 \leq \varphi \leq 2 \pi
\end{aligned}
$$

The prescribed potential distribution along the boundary is $u=x_{1}^{2}-x_{3}^{2}+x_{1} x_{2}+x_{2} x_{3}+2$.

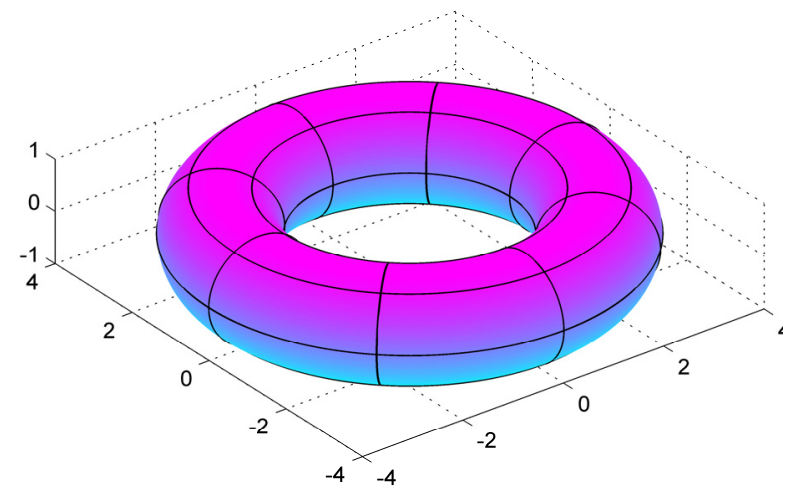

Figure 2: Discretization of the torus with 80 second-order surface elements.

To solve this problem numerically the boundary is discretized by eighty second-order quadrilateral surface elements. Near each boundary element, one interior point is chosen, and thus a total of $N=80$ interior points are taken into account. The average relative error curves of the computational results for the potentials $u$ and its partial derivatives $\partial u / \partial x_{1}$ at these points are shown in Figs 3 and 4, respectively. Hence it can be seen that when the evaluation points 
are not too close to the integration element $(d=0.1)$, the conventional method and the proposed method are both efficient, but the conventional method fails as the evaluation points are closer to the boundary. On the other hand, the results obtained by the proposed method are stable and satisfactory even when the distance of the evaluation point to the integration element is equal to $1.0 \mathrm{E}-9$ or even smaller.

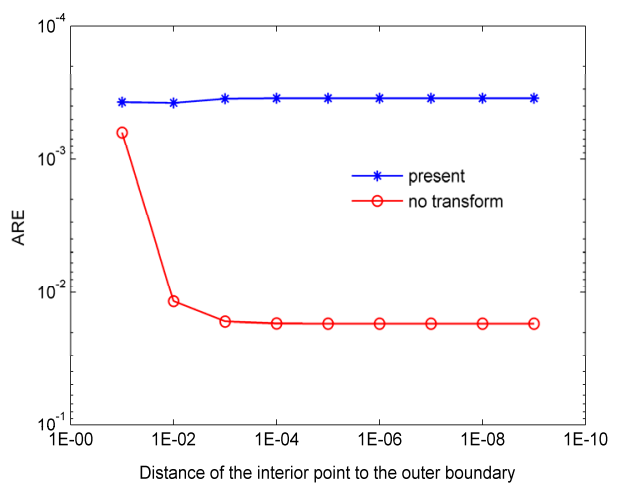

Figure 3: AREs of the potentials $u$ at interior points approaching to the boundary.

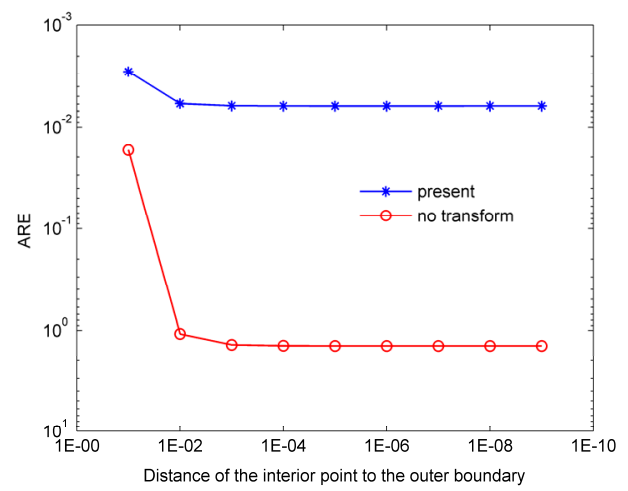

Figure 4: AREs of the fluxes $\partial u / \partial x_{1}$ at interior points approaching the boundary.

\section{Conclusions}

In this paper, a general scheme is proposed in order to calculate the nearly singular integrals occurring on high-order geometrical elements, which arise in the regularized indirect BIEs formulation. The new distance function $r^{2}$ is constructed when the high-order surface elements are adopted for modeling the 
boundary geometry, and then a nonlinear transformation, based on the exponential functions, is introduced to remove the near singularities of the integrals before applying the standard Gaussian quadrature to numerical integration. Three numerical examples with exact benchmark solutions are presented to test the proposed scheme, yielding very promising results even when the distance between the evaluation point and the integration element is as small as $1.0 \mathrm{E}-9$. The results verify the feasibility and the effectiveness of the proposed scheme, with eliminating the boundary layer effect in the regularized BEM formulation.

Compared with existing approaches, the presented scheme makes the first attempt to evaluate the nearly singular integrals arising in the regularized indirect BEM formulations when the high-order quadrilateral surface elements are used.

The proposed scheme also can be applied to other problems in BEM, such as sensitivity analyses, contact problems, and thin-body problems. Some work for thin structures is already underway and will be reported in a subsequent paper.

\section{References}

[1] Tanaka M., Sladek V., Sladek J., Regularization techniques applied to boundary element methods. Applied Mechanics Reviews, 47, pp. 457-499, 1994.

[2] Sladek V., Sladek J., Singular integrals and boundary elements. Computer Methods in Applied Mechanics and Engineering, 157(3-4), pp. 251-266, 1998.

[3] Gao X.W., An effective method for numerical evaluation of general 2D and 3D high order singular boundary elements. Comput Methods Appl. Mech. Engrg, 199, pp. 2856-2864, 2010.

[4] Lachat J.C., Watson JO., Effective numerical treatment of boundary integral equation, a formulation for three-Dimensional elastostatics. International Journal for Numerical Methods in Engineering, 10(5), pp. 991-1005, 1976.

[5] Sladek V., Sladek J., Tanaka M, Regularization of hypersingular and nearly singular integrals in the potential theory and elasticity. International Journal for Numerical Methods in Engineering, 36(10), pp. 1609-1628, 1993.

[6] Gao X.W., Yang K., Wang J., An adaptive element subdivision technique for evaluation of various 2D singular boundary integrals. Engineering Analysis with Boundary Elements, 32, pp. 692-696, 2008.

[7] Ma L.F., Korsunsky A.M., A note on the Gauss-Jacobi quadrature formulae for singular integral equations of the second kind. International Journal of Fracture, 126, pp. 339-405, 2004.

[8] Schulz H., Schwab C., Wendl W.L., The computation of potentials near and on the boundary by an extraction technique for boundary element methods. Computer Methods in Applied Mechanics and Engineering, 157(3-4), pp. 225-238, 1998. 
[9] Liu Y.J., Analysis of shell-like structures by the boundary element method based on 3-D elasticity, formulation and verification. International Journal for Numerical Methods in Engineering, 41(3), pp. 541-558, 1998.

[10] Telles, JCF., A self-adaptive coordinate transformation for efficient numerical evaluation of general boundary element integral. International Journal for Numerical Methods in Engineering, 24, pp. 959-973, 1987.

[11] Cerrolaza M., Alarcon E., A bi-cubic transformation for the numerical evaluation of the Cauchy principal value integrals in boundary methods. International Journal for Numerical Methods in Engineering, 28(5), pp. 987-999, 1989.

[12] Johnston P.R., Application of Sigmoidal transformations to weakly singular and near singular boundary element integrals. International Journal for Numerical Methods in Engineering, 45(10), pp. 1333-1348, 1999.

[13] Johnston P.R., Semi-Sigmoidal transformations for evaluating weakly singular boundary element integrals. International Journal for Numerical Methods in Engineering, 47(10), pp. 1709-1730, 2000.

[14] Sladek V., Sladek J., Tanaka M., Optimal transformations of the integration variables in computation of singular integrals in BEM. International Journal for Numerical Methods in Engineering, 47(7), pp. 1263-1283, 2000.

[15] Lutz E.L., A mapping method for numerical evaluation of two-dimensional integrals with singularity. Computational Mechanics, 12(1-2), pp. 19-26, 1993.

[16] Huang Q., Cruse T.A., Some notes on singular integral techniques in boundary element analysis. International Journal for Numerical Methods in Engineering, 36(15), pp. 2643-2659, 1993.

[17] Ma H., Kamiya N., Distance transformation for the numerical evaluation of near singular boundary integrals with various kernels in boundary element method. Engineering Analysis with Boundary Elements, 26(4), pp. 329339, 2002.

[18] Peter R. Johnston1, David Elliott, A sinh transformation for evaluating nearly singular boundary element integrals. Int. J. Numer. Meth. Engng, 62, pp. 564-578, 2005.

[19] Zhang Y.M., Gu Y., Chen J.T., Boundary layer effect in BEM with high order geometry elements using transformation. Computer Modeling in Engineering and Sciences, 45(3), pp. 227-247, 2009. 